Pacific Journal of Mathematics

MINIMUM PROBLEMS OF PLATEAU TYPE IN THE 


\title{
MINIMUM PROBLEMS OF PLATEAU TYPE IN THE BERGMAN METRIC SPACE
}

\author{
KYONG T. HAHN
}

\begin{abstract}
Dedicated to my teacher Professor C. Loewner on his seventieth birthday
\end{abstract}
1. Introduction. In this paper we are concerned with the existence of minimal surfaces with respect to the $B$-area (see $\S 4$ ) and related problems in a bounded domain $D$ in the space $C^{2}$ of two complex variables $z_{1}, z_{2}$.

Let $\boldsymbol{K}_{D}(z, \bar{z}), z=\left(z_{1}, \cdots, z_{n}\right)$, be the Bergman kernel function of a bounded domain $D$ in the space $C^{n}$ of $n$ complex variables. Throughout this paper, we assume $\boldsymbol{K}_{D}(z, \bar{z})$ has the boundary value infinity at every point on the boundary of $D$. The kernel $\boldsymbol{K}_{D}(z, \bar{z})$ enables us to define the Bergman metric

$$
d s_{D}^{2}(z)=\sum_{\mu, \nu=1}^{n} T_{\mu \bar{\nu}}(z, \bar{z}) d z_{\mu} d \bar{z}_{\nu}, T_{\mu \bar{\nu}}=\frac{\partial^{2} \log \boldsymbol{K}_{D}}{\partial z_{\mu} \partial \bar{z}_{\nu}}
$$

which is invariant with respect to pseudo-conformal mappings [4, pp. 51-53]. Using (1.1) we construct (see §2) the complete Bergman metric space $(D, d)$ over $D$ and state a theorem for complete Riemannian spaces that for any two points in $D$, there exists a minimal curve with respect to $d$ which connects the two points.

In $\S 3$ we show that, if $D$ is a plane domain bounded by finitely many boundary components $b_{1}, b_{2}, \cdots, b_{n}$, then there exists a minimal closed curve with respect to $d$ among those curves which are homotopic to a fixed inner boundary component, say $b_{1}$, in $\overline{D\left(b_{1}\right)}$ (see $\S 3$ for notation). If $D$ is doubly connected, there exists a unique minimal closed curve in $D$. Furthermore, we prove a distortion theorem which gives bounds for the Bergman lengths of the minimal closed curves.

Analogous results are obtained in the case of two complex variables replacing the length by the $B$-area.

For a closed Jordan curve $\Gamma$ in a complete metric space $(D, d)$, we ask whether there exists a minimal surface with respect to the $B$-area which spans $\Gamma$. Answers to this question which constitute the main result of this paper are given in $\S 4$.

As a generalization of $\S 3$, we consider a domain $D$ which is topologically equivalent to a product domain of the form $D_{1} \times D_{2}$,

Received July 5, 1963. This paper represents the essential contents of the author's doctor thesis at Stanford University. The author wishes to express his thanks to Professor S. Bergman and Professor C. Loewner for their pertinent criticism in the preparation of this paper. This research was supported by Navy Contract Nonr-225(11) and NSF Grant 21344. 
where $D_{k}$ is a bounded domain as considered in $\S 3$. When does there exist a minimal closed surface with respect to the $B$-area among those surfaces which are homotopic to $T_{1}$ in $\overline{D\left(T_{1}\right)}$ (see $\S 5$ for notation)?

Answers are given in $\S 5$. Distortion theorems for the minimal surfaces are given in $\S 6$.

2. The Bergman metric space. A (continuous) curve $c$ in $D$ is said to be regular if it admits a regular (parametric) representation, i.e., there exists a continuously differentiable representation

$$
G \mid I: z_{k}=G_{k}(t), k=1,2, \cdots, n, t \in I=[a, b],
$$

and $d G_{k} / d t$ never vanish simultaneously at any $t \in I$. A curve $c$ in $D$ is said to be piecewise regular if it admits a piecewise regular representation, i.e., there exists a partition $\Delta: a=t_{0}<t_{1}<\cdots t_{m-1}<t_{m}=$ $b$ such that $G \mid\left[t_{k-1}, t_{k}\right]$ is regular for $k=1,2, \cdots, m$.

For a piecewise regular curve $c$ given by (2.1), we define

$$
L_{\nu}(c)=\int_{a}^{b}\left[\sum_{\mu, \nu=1}^{n} T_{\mu,(G(t), \overline{G(t)})} \frac{d G_{\mu}}{d t} \frac{\overline{d G_{\nu}}}{d t}\right]^{1 / 2} d t .
$$

$L_{D}(c)$ is independent of the choice of piecewise regular representations of $c . L_{D}(c)$ will be called the Bergman length of $c$.

For any two points $z^{1}$ and $z^{2}$ in $D$, we define a distance function $d$ by

$$
d\left(z^{1}, z^{2}\right)=\inf _{c} L_{D}(c),
$$

where $c$ runs over all piecewise regular curves which connect $z^{1}$ and $z^{2}$. Then the following theorem holds $[15, \S 16]$.

THEOREM 2.1. $d$ satisfies all the axioms for a metric and the metric space $(D, d)$ is topologically equivalent to the metric space $(D, \rho)$ with the Euclidean metric $\rho$. Moreover, the metric space $(D, d)$ is finitely connected in the sense that every pair of points in $D$ can be connected by a curve of finite Bergman length.

The metric space $(D, d)$ will be called the Bergman metric space over $D$. The significance of this metric space is that all metric properties are invariant under pseudo-conformal mappings.

We define the length (generalized) of a continuous curve $c$ in $D$ in the following way: For a partition $\Delta(I)=\left\{I_{1}, I_{2}, \cdots, I_{m}\right\}, I_{k}=$ $\left[t_{k-1}, t_{k}\right], k=1,2, \cdots, m$, of $I$, we define 


$$
\sigma(G ; \Delta(I))=\sum_{k=1}^{m} \sigma\left(G ; I_{k}\right), \quad \sigma\left(G ; I_{k}\right)=d\left(G\left(t_{k}\right), G\left(t_{k-1}\right)\right) .
$$

Further, we define

$$
\mathscr{L}_{D}(c)=\sup _{\Delta} \sigma(G ; \Delta(I)),
$$

where $\Delta$ runs over all possible partitions of $I$. Then $\mathscr{L}_{D}(c)$ is independent of the choice of continuous representations of $c$. Clearly, the functional $\mathscr{L}_{D}$ is lower semi-continuous, i.e.,

$$
\mathscr{L}_{D}(c) \leqq \lim _{k \rightarrow \infty} \inf \mathscr{L}_{D}\left(c_{k}\right) \text {, if } c_{k} \rightarrow c .
$$

Further, for every piecewise regular curve $c, L_{D}(c)=\mathscr{L}_{D}(c)[15, \S 16]$. If $\mathscr{L}_{D}(c)<\infty, c$ is said to be rectifiable. A curve is said to be completely degenerated if there is a representation $G \mid I$ such that $G$ is constant on $I$. A representation $G \mid I$ is said to be normal if $\mathscr{L}_{D}\left(G ;\left[t, t^{\prime}\right]\right)=t^{\prime}-t$, for $t, t^{\prime} \in I, t<t^{\prime}$.

Let $c$ be a rectifiable curve which is not completely degenerated. Then $c$ admits a normal representation $G \mid\left[0, \mathscr{L}_{D}(c)\right]$, If we set $F(t)=$ $G\left(t \mathscr{L}_{D}(c)\right), t \in I_{0}, I_{0}=[0,1]$, then $F \mid I_{0}$ is also a representation of $c$. Such a representation $F \mid I_{0}$ is called a reduced representation of $c$. For a closed curve, $F$ is defined on $(-\infty, \infty)$ and is periodic of period 1. It is, therefore, enough to consider $F$ on $I_{0}$. If $F \mid I_{0}$ is a reduced representation of a curve $c$, then the inequality

$$
d\left(F(t), F\left(t^{\prime}\right)\right) \leqq \mathscr{L}_{D}(c)\left|t-t^{\prime}\right|
$$

holds for every $t, t^{\prime} \in I_{0}$.

A metric space is called complete if every bounded infinite subset contains a limit point in the metric space. If $D$ is homogeneous, $(D, d)$ is always complete. Further, for every bounded generalized analytic polyhedron $D,(D, d)$ is complete. This is a result of $S$. Kobayashi (see [11] for details). For domains $D$ in the space $C^{n}$, $n \leqq 2$, Bergman has shown that the distance from a point in $D$ to the boundary becomes infinite under certain hypothesis on the boundary of $D[1]$, [6, Chap. III]. It is clear, in this case, that the metric space $(D, d)$ is complete. Without going into great details in this direction, we shall assume in the sequel that the metric space $(D, d)$ is always complete.

A curve $K$ in $(D, d)$ which connects $z^{1}$ and $z^{2}$ in $D$ is called a minimal curve between $z^{1}$ and $z^{2}$ if $\mathscr{L}_{D}(K) \leqq \mathscr{L}_{D}(c)$ for all curves $c$ connecting $z^{1}$ and $z^{2}$.

THEOREM 2.2. For any two points $z^{1}$ and $z^{2}, z^{1} \neq z_{2}$, in $(D, d)$, there exists a minimal curve $K$ between $z^{1}$ and $z^{2}$. Further, the 
minimal curves are analytic (see [10] or [15, §17]).

3. The existence of a minimal closed curve in a plane domain and its distortion theorem. We consider a multiply connected bounded domain $D$ in the space $C^{1}$ bounded by $N$ Jordan closed curves $b_{1}, b_{2}, \cdots, b_{n}$, where $b_{n}$ is the outer boundary component. Let $(D, d)$ be the Bergman metric space derived from the Bergman metric

$$
d s_{D}^{2}(z)=\boldsymbol{K}_{D}(z, \bar{z})|d z|^{2} .
$$

It is assumed that $(D, d)$ is complete. Then all the previous considerations, lemmas and theorems can be carried over to this case. We fix an inner boundary component of $D$, say $b_{1}$. Without loss of generality, we may assume $b_{1}$ to be a circle.

Let $\AA\left(D ; b_{1}\right)$ be the class of all closed continuous curves $c$ in $D$ which are homotopic to $b_{1}$ in $\overline{D\left(b_{1}\right)}$, where $D\left(b_{1}\right)$ is a ring domain bounded by $b_{1}$ and $b_{n}$ which contains the domain $D$, and $\overline{D\left(b_{1}\right)}$ is the closure of $D\left(b_{1}\right)$. A curve $K\left(D ; b_{1}\right)$ in $\Re\left(D ; b_{1}\right)$ which satisfies the condition $\mathscr{S}_{D}\left(K\left(D ; b_{1}\right)\right) \leqq \mathscr{L}_{D}(c)$ for all $c \in \Re\left(D ; b_{1}\right)$, will be called a minimal closed curve of $D$ with respect to $b_{1}$. Due to the completeness of $(D ; d)$ and the behavior of $K_{D}(z, \bar{z})$ (described on page 943$)$ on the boundary of $D$, we have

THEOREM 3.1. There exists a minimal closed curve $K\left(D ; b_{1}\right)$ of the domain $D$ with respect to $b_{1}$. Further, it is analytic.

Proof. Let $\gamma=\inf _{c} \mathscr{L}_{D}(c)$, where $c$ runs over the class $\mathscr{R}\left(D ; b_{1}\right)$. Then $0<\gamma<\infty$. There exists a minimizing sequence $\left\{c_{k}\right\}$ of rectifiable curves in $\Re\left(D ; b_{1}\right)$. Let $G_{k} \mid I_{0}$ be the reduced representation of $c_{k}$. By (2.4), we have

$$
d\left(G_{k}(t), G_{k}\left(t^{\prime}\right)\right) \leqq \mathscr{L}_{D}\left(c_{k}\right)\left|t-t^{\prime}\right| \text { for each } k,
$$

and $\left\{\mathscr{L}_{D}\left(c_{k}\right)\right\}$ has an upper bound $\delta$ which is finite. We choose an $M$ such that $M^{1 / 2}>\delta / l\left(b_{1}\right), l\left(b_{1}\right)$ is the Euclidean length of $b_{1}$. Then no $c_{k}$ lies completely in $D-D_{M}, D_{M}=\left[z \mid K_{D}(z, \bar{z}) \leqq M\right]$. Let

$$
\rho=\max _{z_{1} z_{2} \in D_{M}} d\left(z_{1}, z_{2}\right),
$$

then for every pair of positive integers $p$ and $q$, we have $d\left(G_{p}(t), G_{q}(t)\right)<\rho+2 \delta, 0 \leqq t \leqq 1$. Hence, we can select a subsequence $\left\{G_{k_{i}}\right\}$ of $\left\{G_{k}\right\}$ which converges uniformly to a continuous function $G^{0}$ on $I_{0}$. Let $K$ be the closed curve whose representation is given by $G^{0} \mid I_{0}$. Since $c_{k_{i}} \rightarrow K$, and by the lower semi-continuity of $\mathscr{L}_{D}$, we obtain $\mathscr{L}_{D}(K)=\gamma$. The analyticity of $K$ is obvious. 
THEOREM 3.2. Every doubly connected domain has a unique minimal closed curve. It is analytic.

Proof. We shall show first that annulus $Q=[z|r<| z \mid<1]$ has a unique minimal closed curve given by $c_{0}=\left[z|| z \mid=r^{1 / 2}\right]$. Let $P_{1}=$ $\left[z|r<| z \mid<r^{1 / 2}\right], P_{2}=\left[z\left|r^{1 / 2}<\right| z \mid<1\right]$. If $c \cap P_{1}=\phi$, it is immediate that $L_{Q}\left(c_{0}\right) \leqq L_{Q}(c)$, since the kernel function $K_{Q}(z, \bar{z})^{1}$ assumes its minimum on $c_{0}$. If $c \cap P_{2}=\phi$, by the conformal mapping $\zeta=r / z$, we have $\widetilde{c} \cap P_{1}=\phi$, where $\widetilde{c}$ is the image curve of $c$ under $\zeta=r / z$. Since $L_{Q}(\widetilde{c})=L_{Q}(c), L_{Q}\left(c_{0}\right) \leqq L_{Q}(c)$ follows. If $c \cap P_{1} \neq \phi$ and $c \cap P_{2} \neq \phi$, we obtain two closed curves $c_{1}, c_{2}$ consisting of the subarcs of $c$ and $c_{0}$ and such that $c_{1} \cap P_{2}=\phi, c_{2} \cap P_{1}=\phi$. By the previous arguments, $L_{Q}\left(c_{i}\right) \geqq L_{Q}\left(c_{0}\right), \quad i=1,2$. Since $L_{Q}\left(c_{1}\right)+L_{Q}\left(c_{2}\right)=L_{Q}(c)+L_{Q}\left(c_{0}\right)$, we have $L_{Q}\left(c_{0}\right) \leqq L_{Q}(c)$. Let $D$ be a doubly connected domain. Then $D^{\prime}$ can be mapped by a univalent analytic function $f(z)$ onto $Q$. It is clear that $f^{-1}\left(c_{0}\right)$ is the unique minimal closed curve of $D$ with respect. to the inner boundary component by the univalency of $f(z)$.

We consider a domain $D$ in the $z$-plane which is bounded by $b_{1}=[z|| z \mid=r], b_{N}=[z|| z \mid=1]$, and $(N-2)$ closed Jordan curves $b_{2}, \cdots, b_{N-1}$. The curves $b_{2}, \cdots, b_{n-1}$ lie in the domain bounded by $b_{1}$ and $b_{N}$.

Let $A_{1}=[z|r<| z \mid<1], A_{2}=[z|| z-a|<\rho,| z \mid>r]^{2}$, be exterior and interior domains of comparison for $D$, respectively, i.e., $A_{1} \supset D \supset A_{2}$. Then

$$
L_{A_{1}}\left(K\left(A_{1}\right)\right) \leqq L_{l}(K(D)) \leqq L_{A_{2}}\left(K\left(A_{2}\right)\right)
$$

where $K\left(A_{1}\right), K\left(A_{2}\right)$ and $K(D)$ are minimal closed curves of $A_{1}, A_{2}$ and' $D$ with respect to $b_{1}$, respectively. It is an immediate consequence of the fact that if $B \subset A$, then $\boldsymbol{K}_{B}(z, \bar{z}) \geqq \boldsymbol{K}_{\boldsymbol{A}}(z, \bar{z})$ for $z \in B$. The linear transformation

$$
w=\frac{z-(a+\rho d)}{\rho-d(z-a)}, \quad 0<|a| \leqq \rho-r,
$$

maps $A$ onto $Q_{R}=[z|R<| z \mid<1]$, where $R$ is given by

1 A simple computation shows that the kernel function of $Q$,

$$
\boldsymbol{K}_{Q}(z, z)=\frac{1}{\pi|z|^{2}}\left[\mathfrak{P}(2 \log |z| ;-2 \log r, 2 \pi i)+\frac{\zeta(\pi i ;-2 \log r, 2 \pi i)}{\pi i}\right]
$$

(see [9], [18]), where $\mathfrak{P}$ and $\zeta$ are the Weierstrass elliptic functions, assumes its: minimum on $\mathrm{c}_{0}$.

${ }^{2}$ Here we choose $a$ and $\rho$ in such a way that $|z-a|<\rho$ contains $b_{1}$ but no other $b_{k}, k=2, \cdots, N$, and $A_{2}$ to be the largest among such domains. 


$$
\begin{aligned}
& R=\left[\frac{r^{2}-(a+\rho d)^{2}}{(\rho+a d)^{2}-r^{2} d^{2}}\right]^{1 / 2}, \\
& d=\frac{r^{2}-a^{2}-\rho^{2}+\left[\left(r^{2}-a^{2}-\rho^{2}\right)^{2}-4 a^{2} \rho^{2}\right]^{1 / 2}}{2 a \rho} .
\end{aligned}
$$

Since $L_{A_{2}}\left(K\left(A_{2}\right)\right)=L_{Q_{R}}\left(K\left(Q_{R}\right)\right)$, using (3.2), we obtain

THEOREM 3.3. $E(r) \leqq(1 / 2) L_{D}(K(D)) \leqq E(R)$, where $R$ is given by (3.4) and

$$
E(r)=[\pi \mathfrak{s}(\log r ;-2 \log r, 2 \pi i)-i \zeta(\pi i ;-2 \log r, 2 \pi i)]^{1 / 2},
$$

$\mathfrak{P}$ and $\zeta$ are the Weierstrass elliptic functions.

The estimation of the bounds for the Bergman lengths of the minimal closed curves in Theorem 3.3 seems to be done only for a special domain. However, every multiply connected domain can always be mapped onto such a domain by a conformal mapping. Therefore, if we know the geometry of a given domain $D$, combining the various distortion theorems in the theory of conformal mappings and the result in Theorem 3.3, we can obtain various bounds for the Bergman lengths of the minimal curves for quite general domains.

4. The existence of a minimal surface which spans a given closed curve in $(D, d)$. A surface $S$ in the space $C^{2}$ is said to be continuously differentiable if it admits a continuously differentiable representation

$$
G \mid Q_{0}: z_{k}=G_{k}\left(u_{1}, u_{2}\right), k=1,2,\left(u_{1}, u_{2}\right) \in Q_{0}=\left[0 \leqq u_{1}, u_{2} \leqq 1\right] .
$$

A surface $S$ is said to be piecewise continuously differentiable if it admits a piecewise continuously differentiable representation $G \mid Q_{0}$, i.e., there exists a partition $\Delta=\left\{\Delta_{1}, \Delta_{2}, \cdots, \Delta_{m}\right\}$ of $Q_{0}$ by rectilinear triangles $\Delta_{k}$ such that $G \mid \Delta_{k}$ is continuously differentiable, $k=1,2, \cdots, m$. The ordinary $B$-area element at a point $\left(z_{1}, z_{2}\right)$ on a piecewise continuously differentiable surface $S$ is defined by the equation [6, Chap. XI]

$$
d b_{S}(z)=\left|\frac{\partial\left(G_{1}, G_{2}\right)}{\partial\left(u_{1}, u_{2}\right)}\right| d u_{1} d u_{2}
$$

The ordinary area element of $S$ is given by the equation

$$
\begin{aligned}
d a_{S}(z) & =\left[g_{11} g_{22}-\left(R e g_{12}\right)^{2}\right]^{1 / 2} d u_{1} d u_{2}, \\
g_{\alpha \beta} & =\sum_{i=1}^{2} \frac{\partial G_{i}}{\partial u_{\alpha}} \frac{\partial G_{i}}{\partial u_{\beta}}, \alpha, \beta=1,2 .
\end{aligned}
$$

Further (4.1) can also be written in the following form, 


$$
d b_{S}(z)=\left[g_{11} g_{22}-\left|g_{12}\right|^{2}\right]^{1 / 2} d u_{1} d u_{2} .
$$

Therefore, $d a_{S}(z) \geqq d b_{S}(z)$ at every point $z \in S$; the equality holds if and only if $\operatorname{Im} g_{12}=0$.

For a piecewise continuously differentiable surface $S$, the ordinary $B$-area is defined and given by the equation

$$
b(S)=\iint_{Q_{0}}\left|\frac{\partial\left(G_{1}, G_{2}\right)}{\partial\left(u_{1}, u_{2}\right)}\right| d u_{1} d u_{2} .
$$

$b(S)$ is independent of the choice of piecewise continuously differentiable representations $G \mid Q_{0}$ of $S$. $A$ surface $S$ is said to be analytic if it admits an analytic representation $G \mid Q_{0}$, i.e., $\partial G_{k} / \partial \bar{w}=0, k=1,2$, $w=u_{1}+i u_{2}$.

For an analytic or an anti-analytic surface $S, b(S)=0$. It is also clear that $b(S)=0$ if and only if the tangent plane of $S$ at every point is an analytic plane. A simple computation shows the following lemma:

LEMMA 4.1. The following three conditions are equivalent:

1) $b(S)=a(S)$,

2) $\frac{\partial\left(G_{1}, \bar{G}_{1}\right)}{\partial\left(u_{1}, u_{2}\right)}+\frac{\partial\left(G_{2}, \bar{G}_{2}\right)}{\partial\left(u_{1}, u_{2}\right)}=0$ at each point on $S$,

3) $\oint_{c} \bar{G}_{1} d G_{1}+\bar{G}_{2} d G_{2}=0$ for every closed curve $c$ on $S$.

Let $D$ be a bounded domain in the space $C^{2}$ on which $(D, d)$ is complete. The quantity

$$
d B_{D}(z)=\left[K_{D}(z, \bar{z})\right]^{1 / 2} d b_{S}(z), z=\left(z_{1}, z_{2}\right),
$$

is invariant with respect to pseudo-conformal mappings and a monotone decreasing functional of $D[6] . \quad d B_{D}(z)$ is called the invariant $B$-area element of $S$. For a piecewise continuously differentiable surface $S$ in $D$, the invariant $B$-area of $S$ is defined and given by the equation

$$
B_{D}(S)=\iint_{Q_{0}}\left[\boldsymbol{K}_{D}(G, \bar{G})\right]^{1 / 2}\left|\frac{\partial\left(G_{1}, G_{2}\right)}{\partial\left(u_{1}, u_{2}\right)}\right| d u_{1} d u_{2}
$$

and is independent of the choice of piecewise continuously differentiable representations $G \mid Q_{0}$ of $S$.

A surface $S$ in $D$ is said to satisfy the condition $(L)$ with respect to the metric $d$ if there exists a representation $G \mid Q_{0}$ of $S$ for which there exists a constant $L(S)>0$ depending only on $S$ and satisfying the inequality

$$
\mathscr{L}_{D}\left(G ; \sigma\left(w_{1}, w_{2}\right)\right) \leqq L(S)\left|w_{1}-w_{2}\right|
$$


for every pair of points $w_{1} ; w_{2}$ in $Q_{0}$; here $\sigma\left(w_{1}, w_{2}\right)$ is the line segment that joins $w_{1}$ and $w_{2}$ in $Q_{0}, w_{k}=u_{1}^{(k)}+i u_{2}^{(k)}, k=1,2$.

It is clear that $G \mid \partial\left(Q_{0}\right)$, where $\partial\left(Q_{0}\right)$ is the boundary of $Q_{0}$, is a representation of the boundary curve $\Gamma$ of $S$ and that $\Gamma$ is rectifiable. It is also clear that every continuously differentiable surface $S$ satisfies the condition $(L)$ with respect to $d$.

We shall say that a surface $S$ is of class $C^{\prime} \Re(L, N, \Gamma)$ if $S$ admits a continuously differentiable representation

$$
G \mid Q_{0}: z_{l}=G_{k}(w), \quad k=1,2, w \in Q_{0},
$$

which satisfies the following conditions:

(a) for a fixed positive constant $L, L(S) \leqq L$,

(b) for a fixed positive constant $N$,

$$
\left|\frac{\partial G\left(w_{1}\right)}{\partial u_{j}}-\frac{\partial G\left(w_{2}\right)}{\partial u_{j}}\right| \leqq N\left|w_{1}-w_{2}\right|, \quad j=1,2, G=\left(G_{1}, G_{2}\right),
$$

for every pair of points $w_{1}, w_{2}$ in $Q_{0}$,

(c) $S$ spans a preassigned closed Jordan curve $\Gamma$ in $D$ in such a way that $G$ is a one-to-one mapping on $\partial\left(Q_{0}\right)$.

A surface $S_{m}$ is called minimal surface of the class $C^{\prime} \Re(L, N, \Gamma)$ if $B_{D}\left(S_{m}\right) \leqq B_{D}(S)$ for all $S \in C^{\prime} \Re(L, N, \Gamma)$.

THEOREM 4.1.3 For each $L$ and $N$ for which the class $C^{\prime} \Re(L, N, \Gamma)$ is not empty, there exists a minimal surface $S_{m}$ in the class.

Proof. Let $\inf _{S} B_{D}(S)=\gamma$, where $S$ runs over all surfaces in $C^{\prime} \Re(L, N, \Gamma)$. Then $0 \leqq \gamma<\infty$. Hence, there exists a minimizing sequence $\left\{S_{n}\right\}$. Let $G^{n} \mid Q_{0}$ be a representation of $S_{n}$ which satisfies conditions (a), (b) and (c). From (a) it follows that for any pair of positive integers $p, q$,

$$
d\left(G^{p}(w), G^{q}(w)\right) \leqq 2-2^{3 / 2} L .
$$

Therefore, $\left\{\mathrm{G}^{n}(w)\right\}$ is equi-bounded. The equi-continuity of $\left\{G^{n}(w)\right\}$ follows from the inequality

$$
d\left(G^{n}(w), G^{n}\left(w^{\prime}\right)\right) \leqq L\left|w-w^{\prime}\right| \quad \text { for any } w, w^{\prime} \in Q_{0} \text { and all } n .
$$

Hence, we can select a subsequence $\left\{G^{m}(w)\right\}$ of $\left\{G^{n}(w)\right\}$ which converges uniformly to a continuous function $G^{0}(w)$ defined in $Q_{0}$. Let $G^{0} \mid Q_{0}$ define a surface $S_{0}$. Then it is clear that $S_{0}$ spans $\Gamma$ in such a way that $G^{0}$ is a one-to-one mapping on $\partial\left(Q_{0}\right)$. The family $\left\{\partial G^{m} / \partial u_{j}\right\}$

${ }^{3}$ Replacing (a) by the condition $\left(\mathrm{a}^{\prime}\right)$ on page 951 , a result similar to Theorem 4.1 can be given (see Corollary 2). 
of continuous functions $\partial G^{m} / \partial u_{j}$ is equi-bounded and equi-continuous by (b) for $j=1,2$. Therefore, we can select a subsequence $\left\{G^{m_{i}}(w)\right\}$ of $\left\{G^{m}(w)\right\}$ which converges uniformly to $G^{0}(w)$ and such that $\left\{\partial G^{m_{i}} / \partial u_{j}\right\}$ converges uniformly to a continuous function $\partial G^{0} / \partial u_{j}$ for $j=1,2$. This implies that $S_{0}$ is a continuously differentiable surface. In order to show $S_{0} \in C^{\prime} \Re(L, N, \Gamma)$, let $c_{m_{i}}$ and $c_{0}$ be the image curves of a line segment $\sigma\left(w_{1}, w_{2}\right)$ which connects two points $w_{1}$ and $w_{2}$ in $Q_{0}$ under $G^{m_{i}}(w)$ and $G^{0}(w)$, respectively. Then $c_{m_{i}}$ converges to $c_{0}$ and, hence, $\lim _{i \rightarrow \infty} L_{D}\left(c_{m_{i}}\right) \geqq L_{D}\left(c_{0}\right)$ by the lower semi-continuity of $L_{D}$. Since $L_{D}\left(c_{m_{i}}\right) \leqq L\left|w_{1}-w_{2}\right|$ for all $m_{i}, L_{D}\left(c_{0}\right) \leqq L\left|w_{1}-w_{2}\right|$. It is clear that $G^{0}(w)$ satisfies (b). Since the functional $B_{D}$ is lower semi-continuous in $C^{\prime} \Re(L, N, \Gamma)$ and $S_{0} \in C^{\prime} \Re(L, N, \Gamma)$, we have $B_{D}\left(S_{0}\right)=\gamma$. Thus $S_{0}$ is a minimal surface in the class $C^{\prime} \Re(L, N, \Gamma)$.

REMARK. In the case that $\Gamma$ lies on an analytic plane $\pi$ and the portion $\tilde{\pi}$ of $\pi \cap \mathrm{D}$ enclosed by $\Gamma$ is simply connected, $\tilde{\pi}$ is a minimal surface of $C^{\prime} \Re(L, N, \Gamma)$ with some $L$ and $N$, and $B_{D}\left(S_{0}\right)=0$. In general, if there exists an analytic surface $S$ in $D$ which spans $\Gamma$, then $S$ is a minimal surface with some $L$ and $N$, and $B_{0}(S)=0$.

Let $C^{\prime} \Omega(N, \Gamma)$ be the class of continuously differentiable surfaces in the space $C^{2}$ which span a preassigned Jordan closed curve $\Gamma$ in $C^{2}$ and satisfy the condition (b). Then (b) implies condition (a) with respect to the Euclidean metric $\rho$ for every surface in $C^{\prime} \Re(N, \Gamma)$. Since $C^{2}$ is complete with respect to $\rho$, the following corollary follows by the same procedure as in Theorem 4.1.

CoRollary 1. In the class $C^{\prime} \Re(N, \Gamma)$, there exists a minimal surface $S_{m}^{\prime}$ in the sense that

$$
b\left(S_{m}^{\prime}\right) \leqq b(S) \text { for all } S \in C^{\prime} \Re(N, \Gamma) .
$$

Let $C^{\prime} \Re_{\alpha}(N, \Gamma)$ be the class of continuously differentiable surfaces $S$ in $D$ which satisfy conditions (b), (c) and

$\left(a^{\prime}\right)$ for a preassigned real number $\alpha, 0 \leqq \alpha \leqq 1$,

$$
\frac{d b_{S}(z)}{d a_{S}(z)} \geqq \alpha \text { at every point } z \in S \text {. }
$$

We notice that the class $C^{\prime} \mathscr{R}_{\alpha}(N, \Gamma)$ is motone decreasing with respect to $\alpha$.

CoRollaRy 2. For a fixed $\alpha>0$ and $N$ for which $C^{\prime} \mathfrak{R}_{\alpha}(N, \Gamma)$ is not empty there exists a minimal surface in the class.

Proof. The $B$-areas $B_{D}\left(S_{n}\right)$ of $S_{n}$ which belong to a minimizing 
sequence $\left\{S_{n}\right\}$ have a fixed uppper bound. Therefore, condition (a') ensures the existence of an $M>0$ such that every $S_{n}$ lies completely in $D_{M}, D_{M}=\left[z \mid K_{D}(z, \bar{z}) \leqq M\right]$. This implies condition (a) with some $L$, which depends on $\alpha$ and $N$. Hence, the corollary follows from the theorem.

5. The existence of minimal closed surfaces in $(D, d)$. Let $D_{k}$ be a domain in the space of one complex variable $z_{k}$ bounded by $n_{k}$ closed curves $b_{1}^{(k)}, b_{2}^{(k)}, \cdots, b_{n_{k}}^{(k)}$. Here $b_{n_{k}}^{(k)}$ is the outer boundary component of $D_{k}$ and $b_{1}^{(k)}$ is an inner boundary component, which is a circle, i.e., $b_{1}^{(k)}=\left[z_{k}|| z_{k} \mid=r_{k}\right]$.

Let $D$ be a domain in the space $C^{2}$ which is topologically equivalent to the product domain $\widetilde{D}=D_{1} \times D_{2}$, and $T_{1}$ the topological image of $\tilde{T}_{1}=b_{1}^{(1)} \times b_{1}^{(2)}$. A surface $S$ in $D$ which is homotopic to $T_{1}$ in $\overline{\nu\left(T_{1}^{\prime}\right)}$, where $D\left(T_{1}\right)$ is the topological image of $\widetilde{D}\left(\widetilde{T}_{1}\right)=D_{1}\left(b_{1}^{(k)}\right) \times D_{2}\left(b_{2}^{(k)}\right)$ (see $\S 3$ for notation), is a closed surface of the torus type and, hence, admits a doubly periodic representation

$$
\begin{aligned}
G \mid R^{2}: z_{k} & =G_{k}\left(u_{1}, u_{2}\right), k=1,2,\left(u_{1}, u_{2}\right) \in R^{2}, \\
R^{2} & =\left(-\infty<u_{1}, u_{2}<+\infty\right),
\end{aligned}
$$

of periods 1. For our purposes, therefore, it is enough to consider $G$ on the unit square $Q_{0}$ as a representation of $S$.

We shall say that a closed surface $S$ is of class $C^{\prime} \Re_{a}\left(N, T_{1}\right)$ if $S$ is homotopic to $T_{1}$ in $\overline{D\left(T_{1}\right)}$ and admits a continuously differentiable representation $G \mid Q_{0}$ satisfying condition $\left(a^{\prime}\right)$ and $(b)$ in $\S 4$. By the same procedure as in Corollary 2 of Theorem 4.1, we can prove the following theorem for any fixed $\alpha>0$.

THEOREM 5.1. For each $N$ for which the class $C^{\prime} \Re_{\alpha}\left(N, T_{1}\right)$ is not empty, there exists a minimal closed surface $S_{m}(D)$ in the class.

Let $D^{\prime} \Re\left(\widetilde{D}, \widetilde{T}_{1}\right)$ be the class of all closed surfaces $S$ of the form $S=c_{1} \times c_{2}$ in $D$, where $c_{k}$ is a piecewise continuously differentiable closed curve in $D_{k}$ which is homotopic to $b_{1}^{(k)}$ in $\overline{D_{k}\left(b_{1}^{(k)}\right)}$. For each $S \in D^{\prime} \Re\left(\widetilde{D}, \widetilde{T}_{1}\right)$, we have $B_{\widetilde{D}}(S)=L_{D_{1}}\left(c_{1}\right) \cdot L_{D_{2}}\left(c_{2}\right)$. It follows from the fact that $\boldsymbol{K}_{\widetilde{D}}(z, \bar{z})=\boldsymbol{K}_{D_{1}}\left(z_{1}, \bar{z}_{1}\right) \cdot \boldsymbol{K}_{D_{2}}\left(z_{2}, \bar{z}_{2}\right)$ [7]. Therefore, the following is an immediate consequence of Theorem 3.1.

THEOREM 5.2. There exists a minimal surface $S_{m}(\widetilde{D})$ of the class $D^{\prime} \Re\left(\widetilde{D}, \widetilde{T}_{1}\right)$. It is given by $K\left(D_{1}\right) \times K\left(D_{2}\right)$, where $K\left(D_{k}\right)$ is a minimal closed curve of $D_{k}$ with respect to $b_{1}^{(k)}$.

Let $A=A_{1} \times A_{2}$, where $A_{k}$ is a doubly connected plane domain in 
the $z_{k}$-plane. Let $D^{\prime} \Re_{1}(A, T)$ be the class of piecewise continuously differentiable closed surfaces $S$ in $A$ which are homotopic to $T=$ $b_{1}^{(1)} \times b_{2}^{(2)}$ in $\bar{A}$, where $b_{1}^{(k)}$ is the inner boundary component of $A_{k}$, and satisfy the condition $d a_{S}(z)=d b_{S}(z){ }^{4}$ Then the following theorem holds:

THEOREM 5.3. There exists a unique minimal closed surface in the class $D^{\prime} \Re_{1}(A, T)$. It is given by $K\left(A_{1}\right) \times K\left(A_{2}\right)$, where $K\left(A_{k}\right)$ is a minimal closed curve of $A_{k}$ with respect to $b_{1}^{(k)}$.

Proof. Let $A=Q=Q_{1} \times Q_{2}, Q_{k}=\left[z_{k}\left|r_{k}<\right| z_{k} \mid<1\right]$. We shall show $S_{m}(Q)=K\left(Q_{1}\right) \times K\left(Q_{2}\right)$ is a unique minimal closed surface of $D^{\prime} \Re_{1}(Q, T)$. Let $P_{1 k}=\left[z_{k}\left|r_{k}<\right| z_{k k} \mid<r_{k}^{1 / 2}\right], \quad P_{2 k}=\left[z_{k}\left|r_{k}^{1 / 2} \leqq\right| z_{k} \mid<1\right]$. If $S \in P_{21} \times P_{22}$, it is immediate that $B_{Q}(S) \geqq B_{Q}\left(S_{m}\right)$. For any $S \in D^{\prime} \Re_{1}(Q, T), S$ can be replaced by a surface $\widetilde{S} \in D^{\prime} \Re_{1}(Q, T)$ with $B_{Q}(S)=B_{Q}(\widetilde{S})$ and lying in $P_{21} \times P_{22}$ by the pseudo-conformal mapping $z_{k}^{*}=r_{k} / z_{k}, k=1,2$. Thus, $B_{Q}(S) \geqq B_{Q}\left(S_{m}\right)$ for every $S \in D^{\prime} \Re_{1}(Q, T)$. There exists a univalent analytic function $f_{k}\left(z_{k}\right)$ which maps $A_{k}$ onto $Q_{k}$. Therefore, the pseudo-conformal mapping $w_{k}=f_{k}^{-1}\left(z_{k}\right)$ maps $A$ onto $Q$ and, hence, $S_{m}(Q)$ onto $S_{m}(A), S_{m}(A)=K\left(A_{1}\right) \times K\left(A_{2}\right)$. The uniqueness of $S_{m}(A)$ is clear.

6. Bounds for the $B$-areas of minimal closed surfaces in the space $(D, d)$. Using the method of exterior and interior domains of comparison, various bounds for the $B$-areas of minimal surfaces can be obtained. As we have considered in $\S 3$, let $D_{k}$ be bounded by $b_{1}^{(k)}=\left[z_{k}|| z_{k} \mid=r_{k}\right], \quad b_{n_{k}}^{(k)}=\left[z_{k}|| z_{k} \mid=1\right]$, and $\left(n_{k}-2\right)$ closed Jordan curves $b_{2}^{(k)}, \cdots, b_{n_{k}-1}^{(k)}$, which lie in the domain bounded by $b_{1}^{(k)}$ and $b_{n_{k}}^{(k)}$. Let $A_{1 k}=\left[z_{k}\left|\boldsymbol{r}_{k}<\right| z_{k} \mid<1\right], \quad A_{2 k}=\left[z_{k}|| z_{k}-a_{k}\left|<\rho_{k},\right| z_{k} \mid>\boldsymbol{r}_{k}\right]$, $0<\left|a_{k}\right| \leqq \rho_{k}-r_{k}$, be exterior and interior domains of comparison for $D_{k}$, respectively. Then $A_{j}=A_{j 1} \times A_{j 2}$ can be used as exterior and interior domains of comparison of $D=D_{1} \times D_{2}$, i.e., $A_{1} \supset D \supset A_{2}$. Let $S_{m}(\widetilde{D})$ and $S_{n i}\left(A_{j}\right)$ be minimal surfaces of the classes $D^{\prime} \Re\left(\widetilde{D}, \widetilde{T}_{1}\right)$ and $D^{\prime} \Re\left(A_{j}, \widetilde{T}_{1}\right)$, respectively. Then $B_{A_{1}}\left(S_{m}\left(A_{1}\right)\right) \leqq B_{\widetilde{D}}\left(S_{m}(D)\right) \leqq B_{A_{2}}\left(S_{m}\left(A_{2}\right)\right)$. Using this inequality, we have the following distortion theorem for minimal surfaces of the class $D^{\prime} \Re\left(\widetilde{D}, \widetilde{T}_{1}\right)$.

THEOREM 6.1. $\prod_{k=1}^{2} E\left(r_{k}\right) \leqq(1 / 4) B_{\widetilde{\nu}}\left(S_{m}\left(\widetilde{D}, \widetilde{T}_{1}\right)\right) \leqq \prod_{k=1}^{2} E\left(R_{k}\right)$, where $R_{k}$ is given in (3.4) with the corresponding subscript $k$ and $E(r)$ is given in Theorem 3.3.

By a construction of an interior domain of comparison for $D$ in

${ }_{4}^{4}$ This is the case $\alpha=1$ in $\left(a^{\prime}\right)$ (see (4.8) and Lemma 4.1). 
Theorem 5.1, we can also obtain a distortion theorem for minimal surfaces $S_{m}(D)$ in Theorem 5.1 which gives us an upper bound. Suppose an interior domain of comparison for $D$ is given by $A_{2 k}$, then we have

THEOREM 6.2. $\quad B_{D}\left(S_{m}(D)\right) \leqq 4 \prod_{k=1}^{2} E\left(R_{k}\right)$, where $R_{k}$ and $E(r)$ are given as in Theorem 6.1.

REMARK. For the product domain $Q=Q_{1} \times Q_{2}$ of two annuli $Q_{1}$ and $Q_{2}, K\left(Q_{1}\right) \times K\left(Q_{2}\right)$ is not necessarily a minimal surface for the class $D^{\prime} \Re_{\alpha}(Q, T)$ for a fixed $\alpha, 0<\alpha<1$.

\section{BIBLIOGRAPHY}

1. S. Bergman, Über die Kernfunktion eines Bereiches und ihr Verhalten am Rande, J. Reine Angew. Math., 169 (1933), 1-42, and 172 (1934), 89-128.

2. - Sur quelques propriétés des transformations par un couple de fonctions de deux variables complexes, Rend. Della R. Accad. Naz. Del Lincei, 19 (ser. 6) (1934), 474-478.

3. - Zur Theorie von Pseudokonformen Abbildungen, Recueil Math., nouv., 1 (43), (1936), 76-96.

4. - Sur les functions orthogonales de plusieurs variables complexes, Mém. des Sciences Math., 106 (1947).

5. — Sur la fonction-noyau d'un domaine et ses applications dans la théorie des transformations pseudo-conformes, Mém. des Sciences Math., 108 (1948).

6. - The kernel function and conformal mapping, Math. Surveys 5, Amer. Math. Soc., New York, 1950.

7. H. J. Bremermann, Holomorphic continuation of the kernel function and the Bergman metric in several complex variables, in Lectures on functions of a complex variable ed. by W. Kaplan, Univ. of Michigan Press, 1955.

8. H. Busemann, and M. Mayer, On the foundations of calculus of variations, Trans. Amer. Math. Soc., 49 (1941), 173-198.

9. B. Epstein, The kernel-function and conformal invariants, J. Math. and Mech., 7 (1958), 925-936.

10. D. Hilbert, Über das Dirichletsche Princip, Jahr. Dtsch. Math.-Verein., 8 (1900), 184-188.

11. S. Kobayashi, Geometry of Bounded Domains, Trans. Amer. Math. Soc., 92 (1959), :267-290.

12. C. B. Morrey Jr., Quasi-convexity and the lower semi-continuity of multiple integrals, Pacific J. Math., 2, (1952), 25-53.

13. - The parametric variational problem for double integrals, Communications on Pure and Applied Math., 14 (1961), 569-575.

14. K. Reinhardt, Über Abbildungen durch analytische Funktionen zweier Veranderlicher, Math. Ann., 83 (1921), 211-255.

15. W. Rinow, Die Innere Geometrie der Metrischen Räume, Die Grundlehren der Math. Wissenschaften 105 (1961).

16. J. M. Stark, On distortion in pseudo conformal mappings, Pacific J. Math., 6 (1956), $565-582$.

17. - Minimum problems in the theory of pseudo-conformal transformations 
and their application to estimation of the curvature of the invariant metric, Pacific J. Math., 10 (1960), 1021-1038.

18. K. Zarankiewicz, Über ein numerisches Verfahren zur konformen Abbildung zweifach zusammenhangender Gebiede, Zeits. f. ang. Math. u. Mech. 14 (1934), 97-104.

STANFORD UNIVERSITY 



\section{PACIFIC JOURNAL OF MATHEMATICS}

\section{EDITORS}

\author{
Robert Osserman \\ Stanford University \\ Stanford, California
M. G. Arsove
University of Washington
Seattle 5 , Washington

\author{
J. DugundJI \\ University of Southern California \\ Los Angeles 7, California
}

Lowell J. Paige

University of California

Los Angeles 24, California

\section{ASSOCIATE EDITORS}
E. F. BECKENBACH
B. H. NeumanN
F. WOLF
K. YOSIDA

\section{SUPPORTING INSTITUTIONS}

\author{
UNIVERSITY OF BRITISH COLUMBIA \\ CALIFORNIA INSTITUTE OF TECHNOLOGY \\ UNIVERSITY OF CALIFORNIA \\ MONTANA STATE UNIVERSITY \\ UNIVERSITY OF NEVADA \\ NEW MEXICO STATE UNIVERSITY \\ OREGON STATE UNIVERSITY \\ UNIVERSITY OF OREGON \\ OSAKA UNIVERSITY \\ UNIVERSITY OF SOUTHERN CALIFORNIA
}

\author{
STANFORD UNIVERSITY \\ UNIVERSITY OF TOKYO \\ UNIVERSITY OF UTAH \\ WASHINGTON STATE UNIVERSITY \\ UNIVERSITY OF WASHINGTON \\ * * * * \\ AMERICAN MATHEMATICAL SOCIETY \\ CALIFORNIA RESEARCH CORPORATION \\ SPACE TECHNOLOGY LABORATORIES \\ NAVAL ORDNANCE TEST STATION
}

Mathematical papers intended for publication in the Pacific Journal of Mathematics should by typewritten (double spaced), and on submission, must be accompanied by a separate author's résumé. Manuscripts may be sent to any one of the four editors. All other communications to the editors should be addressed to the managing editor, L. J. Paige at the University of California, Los Angeles 24, California.

50 reprints per author of each article are furnished free of charge; additional copies may be obtained at cost in multiples of 50 .

The Pacific Journal of Mathematics is published quarterly, in March, June, September, and December. Effective with Volume 13 the price per volume (4 numbers) is $\$ 18.00$; single issues, $\$ 5.00$. Special price for current issues to individual faculty members of supporting institutions and to individual members of the American Mathematical Society: $\$ 8.00$ per volume; single issues $\$ 2.50$. Back numbers are available.

Subscriptions, orders for back numbers, and changes of address should be sent to Pacific Journal of Mathematics, 103 Highland Boulevard, Berkeley 8, California.

Printed at Kokusai Bunken Insatsusha (International Academic Printing Co., Ltd.), No. 6, 2-chome, Fujimi-cho, Chiyoda-ku, Tokyo, Japan.

PUBLISHED BY PACIFIC JOURNAL OF MATHEMATICS, A NON-PROFIT CORPORATION

The Supporting Institutions listed above contribute to the cost of publication of this Journal, but they are not owners or publishers and have no responsibility for its content or policies. 


\section{Pacific Journal of Mathematics}

\section{Vol. 14, No. $3 \quad$ July, 1964}

Erik Balslev and Theodore William Gamelin, The essential spectrum of a class of ordinary differential operators . . . . . . . . . . . . . . . . . . . .

James Henry Bramble and Lawrence Edward Payne, Bounds for derivatives in

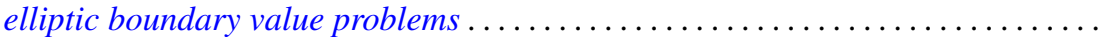

Hugh D. Brunk, Integral inequalities for functions with nondecreasing

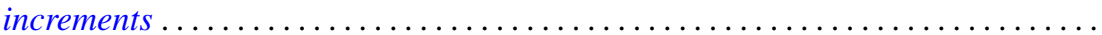

William Edward Christilles, A result concerning integral binary quadratic

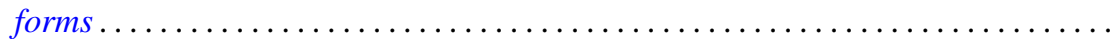

Peter Crawley and Bjarni Jónsson, Refinements for infinite direct decompositions of

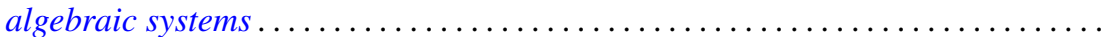

Don Deckard and Carl Mark Pearcy, On continuous matrix-valued functions on a Stonian space.

Raymond Frank Dickman, Leonard Rubin and P. M. Swingle, Another

characterization of the $n$-sphere and related results $\ldots \ldots \ldots \ldots \ldots \ldots$

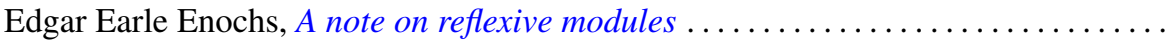

Vladimir Filippenko, On the reflection of harmonic functions and of solutions of the

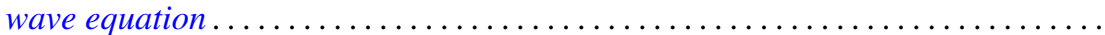

Derek Joseph Haggard Fuller, Mappings of bounded characteristic into arbitrary

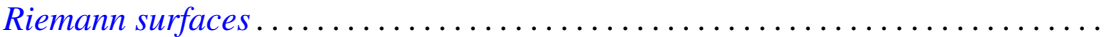
895

Curtis M. Fulton, Clifford vectors . . . . . . . . . . . . . . . . . . . . . . . . . . . . . . 917

Irving Leonard Glicksberg, Maximal algebras and a theorem of Radó . .

919

Kyong Taik Hahn, Minimum problems of Plateau type in the Bergman metric

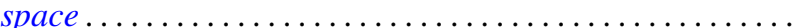

A. Hayes, A representation theory for a class of partially ordered rings...

J. M. C. Joshi, On a generalized Stieltjes trasform

J. M. C. Joshi, Inversion and representation theorems for a generalized Laplace transform ...

Eugene Kay McLachlan, Extremal elements of the convex cone $B_{n}$ of functions ...

Robert Alan Melter, Contributions to Boolean geometry of p-rings ...

James Ronald Retherford, Basic sequences and the Paley-Wiener criterion . . . . . . . 1019

Dallas W. Sasser, Quasi-positive operators. .

Oved Shisha, On the structure of infrapolynomials with prescribed coefficients ..

Oved Shisha and Gerald Thomas Cargo, On comparable means

Maurice Sion, A characterization of weak ${ }^{*}$ convergence ........

Morton Lincoln Slater and Robert James Thompson, A permanent inequality for

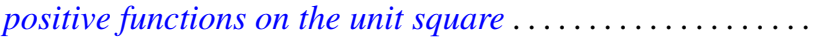

David A. Smith, On fixed points of automorphisms of classical Lie algebras ...

Sherman K. Stein, Homogeneous quasigroups ................

J. L. Walsh and Oved Shisha, On the location of the zeros of some infrapolynomials with prescribed coefficients .

Ronson Joseph Warne, Homomorphisms of $d$-simple inverse semigroups with identity . 